\title{
Aspectos epidemiológicos da fluorose dentária no Brasil: pesquisas no período 1993-2006
}

\author{
Epidemiologic aspects of dental fluorosis in Brazil: \\ research in the period 1993-2006
}

Bruno Salles de Almeida Barros ${ }^{1}$

Nilce Emy Tomita ${ }^{1}$

${ }^{1}$ Departamento de Odontopediatria, Ortodontia eSaúdeColetiva daFaculdadede

Odontologia de Bauru, Universidade de São Paulo. Alameda O ctávio Pinheiro Brisolla 9-75. 17012-901

Bauru SP.netomita@usp.br
Abstract The epidemiology of dental fluorosis de rives from surveys carried out in recent years, as a result of a better comprehension of metabolic aspects of fluoride in the human organism and oral health concerns. This reflection aims at presenting studies carried out on fluorosis between 1993 and 2006. The period of 1993-2004 delimits the interval between the $2^{\text {nd }}$ and the $3^{\text {rd }} \mathrm{National}$ Conferences on Oral $\mathrm{H}$ ealth, and, in the period of 20052006, the search of primary data presented in scientific meetings confirmed the findings in the literature, showing that the Brazilian scientific agenda was not substantially influenced by the discussions engaged during the $3^{\text {rd }} \mathrm{N}$ ational Conference on Oral $\mathrm{H}$ ealth. M ost studies concentrate on urban areasand thepredominance, in Brazil, of "very mild" and "mild" levels of fluorosis shows that there is no compromising in terms of functional order. The low perception of fluorosis by the population, along with its low prevalence, evokes the necessary debate on public health issues, in the country. Since the national scientific production constitutes an important source of knowledge to subsidize the elaboration of public policies for the health sector, the successive and punctual studies analyzed show that, asfar asfluorosis isconcerned, the epidemiological diagnosis reaffirms the need, importance and safety of the fluoridation of public water supplies, as a public health measure. Key words Epidemiology, Dental fluorosis, Children
Resumo A epidemiologia da fluorose dentária re sulta deinquéritos realizados recentemente, em de corrência da melhor compreensão de aspectos me tabólicos dos fluoretos no organismo humano e de preocupações no âmbito da saúde coletiva. Objetiva-se apresentar os estudos realizados sobre a fluorose entre 1993 e 2006. 0 período 1993-2004 demarca o intervalo entrea $2^{\mathrm{a}}$ ea $3^{\mathrm{a}}$ Conferência $\mathrm{Na}$ cional de Saúde Bucal. Em 2005-2006, a busca de dados primários apresentados em reuniões científicas confirmou os achados existentes na literatura, mostrando que a agenda científica brasileira não foi substancialmente influenciada pelas discussões travadas durante a 3a Conferência. A maioria dos estudos concentra-se em áreas urbanas e a predominância no Brasil dos graus "muito leve" e "leve" de fluorose mostra não haver comprometimentos de ordem funcional. A baixa percepção da fluorose pela população, aliada à sua baixa prevalência, evoca - necessário debate sobre as questões de saúde cole tiva no país. Visto que a produção científica nacional constitui uma importante fonte de conhecimentos para subsidiar a elaboração de políticas públicas para o setor da saúde, os sucessivose pontuais estudos analisados mostram que, no que diz respeito à fluorose, o diagnóstico epidemiológico reafirma a necessidade, a importância ea segurança da fluoretação daságuas deabastecimento público como medida de saúde coletiva.

Palavras-chave Epidemiologia, Fluorose dentária, Crianças 
Introdução

$\mathrm{Na}$ atualidade, algumas consequências da chamada "transição epidemiológica" em saúde bucal fazem com que nos deparemos com um quadro simultâneo de redução da cárie dentária em crianças e el evado edentulismo em adultos.

A epidemiologia da fluorose dentária éresultante de inquéritos real izados nos anos recentes, em decorrência da melhor compreensão de aspectos metabólicos dos fluoretos no organismo humano e de preocupações no âmbito da saúde coletiva.

A fluoretação da água vem sendo recomendada pela Organização Mundial de Saúde desde a década de cinquenta, por ser um método eficaz, econômico e abrangente na prevenção da cárie dentária. Entretanto, o flúor ingerido continuamente em excesso podecausar fluorose dentária, a qual é agravada por fatores como o baixo peso corporal, taxa de crescimento esquelético e períodos de remodelamento ósseo.

Revisões sistemáticas e análises críticas permitem uma reflexão quanto ao papel da fluorose no elenco das prioridades em saúde coletiva. A pre sente reflexão tem como objetivo apresentar estudos realizados sobre a fluorose no período 19932006, contribuindo para este importante debate.

\section{Revisão da literatura}

Paiva et al. ${ }^{1}$ avaliaram 299 crianças, de ambos os sexos, entre cinco e dez anos completos, escolares do distrito de Cocal, município de Urussanga (SC). 0 índice defluorose dentária, conforme preconizado por Dean, foi estimado para a dentição permanente. A variação do grau de fluorose mostrou-se independente do sexo da criança, porém ficou constatado um aumento progressivo relacionado ao avanço da idade.

Tomita et al. ${ }^{2}$ realizaram um levantamento epidemiológico nas três escolas públicas do município de Piratininga (SP), o qual apresenta fluoretação das águas de abastecimento realizada por serviço autônomo. Os exames foram realizados por uma única examinadora, em uma amostra de 270 escolares de ambos os sexos, de seis a catorze anos de idade. Em 1991 e 1992, foram coletadas amostras de água das três escolas visitadas, apresentando médias de 1,05 e 0,72 ppm de flúor, respectivamente. 0 resultado do estudo utilizando o índice de fluorose de Dean foi de $27,8 \%$ muito leve, 4,4 leve, $2,2 \%$ moderada e, de acordo com o índice TF, observou-se $20 \%$ de cri- anças com $\mathrm{TF}=1,7,78 \%$ com $\mathrm{TF}=2,4,44 \%$ com $\mathrm{TF}=3,1,85 \%$ com TF $=4$ e $0,37 \%$ com TF $=5$. Devido aos teores deflúor estarem entre 0,7 e1ppm e um terço das crianças apresentarem fluorose dentária, deve-se estimular um maior controle na fluoretação e orientação sobre o uso de produtos fluoretados, por parte de ações de vigilância à saúde.

Campos et al. ${ }^{3}$ examinaram 833 crianças com idade entre oito e doze anos, residentes em Brasília desde o nascimento. Para isso, utilizou-se cadeira comum, o campo foi seco com gaze, sob luz natural, examinando-se todos os dentes superiores permanentes presentes. Foi utilizado o índice TSIF idealizado por H orowitz et al. em 1984. Observou-se em tal exame que apenas $14,64 \%$ das crianças apresentaram algum grau de fluorose, que variou entre 1 a 3, sendo $10,44 \%$ de grau 1 , $3,6 \%$ degrau 2 e $0,6 \%$ degrau 3 . Não foi observada diferença na prevalência entre os sexos.

$M$ endonça et al . ${ }^{4}$ realizaram um estudo transversal em Belo Horizonte (MG), verificando a prevalência da fluorose dentária em 797 escolares de sete e dez anos. Os resultados apresentados referem-seàs crianças de seteanos, sendo a fluorose branda o tipo mais comum. Dentre as crianças que apresentam fluorose $(52,2 \%), 82,7 \%$ apresentam de forma branda, 4,9\%, questionável, e $12,3 \%$, severa. As crianças subnutridas apresentaram mais fluorose que as bem nutridas. A concentração de flúor no ar e a altitude dos setores analisados devem ser verificadas, para que novos parâmetros se estabeleçam para se entender a alta ocorrência de fluorose em Belo H orizonte.

Ribas et al. ${ }^{5}$ avaliaram a ocorrência da fluorose dentária em escolares da rede particular, na região da Penha, Vila Laís, onde se adiciona flúor em níveis ideais nas águas de abastecimento público. A plicou-seo índiceTF no exameclínico, o qual foi realizado com 553 crianças deseisa doze anos. Apresentaram algum grau de fluorose $29,47 \%$ dos escolares, dos quais $71,78 \%$ apresentaram índice $\mathrm{TF}=1,21,47 \%$, índice $\mathrm{TF}=2$ e $6,75 \%$, índice $T F=3$. Na maioria dos casos observados de fluorose dentária (29\% das crianças), foi detectada a forma leve. Em função dos dados obtidos, concluiu-se que os pais e responsáveis devem ser orientados para que acompanhem a utilização dos compostos fluorados, e devem ser feitos estudos visando restabelecer uma dose ideal flúor/dia, com ênfase na obtenção de máxima proteção à cárie dentária e mínimo risco de fluorose dentária.

Dini et al. ${ }^{6}$ observaram a prevalência de cárie dentária, do desenvolvimento de defeitos no es- 
malte e a associação entre eles em crianças de nove e dez anos de idade moradoras em áreas com diferentes níveis de flúor na água de abastecimento. As crianças pertenciam às cidades de AraraquaraeAmérico Brasiliense, com suaságuas fluoretadas desde 1963 e 1998, respectivamente. Um total de 287 crianças de Araraquara e 210 de Américo Brasiliense foram examinadas. As crianças moradoras na área fluoretada desde 1963 que apresentaram apenas opacidades difusas tinham duas vezes mais chances de não apresentarem cárie quando comparadas com aquelas da mesma área que não apresentavam defeitos ou apresentavam defeitos hipoplásicos. Este estudo confirma os benefícios da fluoretação da água em relação à cárie dentária e mostra que opacidades difusas em incisivos superiores afetaram alguns indivíduos nas duas cidades.

Cangussu et al. ${ }^{7}$ realizaram um estudo no município de Itatiba (SP), com águas de abastecimento público com 0,7 miligramas deflúor por litro, auxiliados por cinco dentistas, euma amostra de 109 crianças de cinco anos, 90 de dozeanos e53 adolescentes de quinzeanos. A maior prevalência foi observada nos adolescentes dedozeanos de idade, e nas formas mais leves da doença. A maior ocorrência da fluorose dental em idades mais jovens pode ter relação com o aumento do uso de formas tópicas de flúor nos dentifrícios e enxaguatórios bucais. Foi encontrada menor pre valência de fluorose dental em crianças com menores níveis de renda. Os níveis de severidade não são maiores do que o esperado para um município com fluoretação de suas águas; porém, deve haver um melhor controle na fluoretação em diferentes áreas, visto que al gumas áreas mostraram maiores prevalências de fluorose mais acometidas por esta alteração.

Forte et al. ${ }^{8}$ avaliaram a prevalência de fluorose dentária em escolares de Princesa I sabel, $\mathrm{Pa}$ raíba. Para isto, foram selecionados 142 escolares de dez a quinze anos, de ambos os sexos. Os exames foram realizados por três profissionais pre viamentecalibrados, sob luz natural indireta após escovação supervisionada e secagem dos elementos dentários. Cerca de $20 \%$ dos examinados apresentaram fluorose dentária, sendo $70 \% \mathrm{com}$ TF 1 eos outros $30 \%$ entre os graus 2 e 5 . Foi mais prevalente no sexo masculino e em dentes pré molares. A fluorose dentária observada foi considerada em níveis esperados pelo teor de fluoretos ( 0,4 ppm) encontrado em amostras de água de abastecimento da cidade. Também observouse alguns casos de fluorose com comprometimento estético enecessidade de tratamento.
Oliveira e Milbourne ${ }^{9}$ realizaram um estudo com 266 crianças, com idades entre sete e doze anos, de uma escola pública do município do Rio deJaneiro (RJ). Os examesclínicosforam realizados por um único profissional, utilizando o índice TF. A limpeza e a secagem dos dentes foram realizadas pelas crianças com rolos de algodão. $A$ prevalência de fluorose dentária entre todas as crianças foi de 7,9\%, sendo o grau 3 o maior encontrado, e o mais frequente foi o grau 1 , com $77 \%$ entre os portadores desta alteração. 0 teor deflúor na água do bairro onde foi feita a pesquisa era igual a 0,18ppm. Concluiu-se neste estudo que a fluorose dentária não constitui um problema de saúde pública para a população estudada.

Silva e $M$ altz ${ }^{10}$ avaliaram a prevalência e severidade decárieefluorosedeescolares dedoze anos de Porto Alegre (RS). Foram examinados mil escolares da rede de ensino público e particular da cidade. Osíndicesutilizados foram o CPO eo ITF. As crianças eram submetidas à remoção da placa e secagem dos dentes para a realização dos exames de cárie e fluorose. Não foi observada diferença estatística entre os sexos em nenhum dos aspectos examinados. Os CPOS foram baixos. Em relação à fluorose, em $52,9 \%$ das crianças a adversidade foi detectada, sendo que $45,9 \%$ dos estudantes apresentavam ITF - 1, 6,1\%, ITF - 2 e $0,9 \%$, ITF - 3. Constatou-se um aumento na prevalência de fluorose dentária na última década; entretanto, a severidade da fluorose encontrada não justifica qualquer medida de saúde pública.

Alves et al. ${ }^{11}$ realizaram um estudo em doze escolas da rede pública de M arília (SP), município que tem fluoretação de suas águas na concentração de $0,7 \mathrm{mg} / \mathrm{L}$, sendo examinadas 994 crianças de três a seis anos de idade, de ambos os sexos, a fim de observar a prevalência de fluorose dentária. Foram realizados por acadêmicos de odontologia da UN IM AR, nos pátios das escolas sob luz natural. Utilizou-se o índice de Dean, observando-se o seguinte resultado aos três anos de idade: $2,7 \%$ grau $1 ; 0,9 \%$ grau $2 ; 0,4 \%$ grau 3 ; $0,4 \%$ grau $4 ; 0,4 \%$ grau 5 ; aos quatro anos de idade: $2,2 \%$ grau $1 ; 4,1 \%$ grau $20,4 \%$ grau 3 e nenhuma criança grau 4 e 5 ; aos cinco anos: $4,5 \%$ grau $1 ; 3 \%$ grau $2 ; 1,9 \%$ grau $3 ; 0,8 \%$ grau 4 e nenhuma criança grau 5 ; aos seis anos: $6 \%$ grau 1; $3 \%$ grau 2; $0,9 \%$ grau 3; nenhuma grau $4 \mathrm{e}$ $0,4 \%$ grau 5 . Em função da baixa prevalência de fluorose dentária nos pré-escolares estudados, a mesma não constitui um problema de amplas dimensões para a saúde pública, mesmo tendose observado crianças acometidas por fluorose em graus moderado e severo. 
M enezes et al. ${ }^{12}$ realizaram o trabalho em uma escola pública da cidade de Piracicaba (SP), com 57 alunos de dez a catorze anos de ambos os sexos. Os estudantes estavam diariamente expostos a dentifrício fluoretado, além daágua deabastecimento otimamente fluoretada ( $0,7 \mathrm{ppm}$ de $\mathrm{F}$ ) desde 1971. 0 índice utilizado foi o de Dean. Foi aplicado um questionário semi-estruturado sob supervisão, quando da tomada das fotos, objetivando avaliar se a fluorose dental era percebida por adolescentes e qual o grau de insatisfação com seus dentes. Dos adolescentes entrevistados, $28 \%$ não apresentavam fluorose, $35,1 \%$ apresentavam fluorose questionável, $24,5 \%$, fluorose muito leve e $12,4 \%$, fluorose leve. Constatou-se que a única pessoa que percebeu a mancha nos dentes não apresentava fluorose. Concluiu-se então que há segurança na associação de dentifrício fluoretado com água fluoretada na concentração ótima.

Cypriano et al. ${ }^{13}$ verificaram a prevalência e severidade de fluorose. Foi realizado um levantamento epidemiológico em 2.805 crianças decinco e seis anos de escolas municipais de Piracicaba (SP). 0 delineamento foi do tipo transversal. Quanto à prevalência de fluorose dentária, encontrou-se $2,6 \%$ e $6,1 \%$ aos cinco e seis anos, respectivamente, sendo o grau "muito leve" 0 mais frequente. A fluorose dentária aumentou $3,5 \%$ dos cinco para os seis anos. Apenas $1,4 \%$ das crianças aos cinco anos e 2,1\% aos seis anos apresentaram graus leve e moderado.

Cangussu et al..$^{14}$ encontraram as prevalências de fluorose dentária de $31,4 \%$ aos doze anos de idade, e de $27,6 \%$ aos quinze. 0 estudo foi realizado em uma amostra de 3.313 escolares de doze e quinze anos, em distrito sanitário de Salvador (BA). O bservou-seum predomínio defluorose no grau muito leve. Em diferentes distritos sanitários do município, notaram-se prevalências bem discrepantes, reforçando a necessidade de vigilância à saúde. A fluorose dentária parece não constituir um problema de saúde pública no município.

Balen et al. ${ }^{15}$ investigaram a associação entre creme dental, suplementos de flúor, enxaguatórios que contêm flúor com o índice de fluorose, avaliando sua autopercepção. Foi aplicado um questionário analisando a quantidade e frequência deflúor utilizado pelas crianças e sua autopercepção. Em relação à autopercepção da fluorose, $74,06 \%$ dos casos não notaram manchamento e 22,04\% destas não se incomodam.

Kern et al. ${ }^{16}$ verificou a autopercepção de fluorose dentária e satisfação com a aparência den- tal em crianças de doze anos. A prevalência de fluorose foi de $20,35 \%$, e a severidade foi de $14,16 \%$ muito leve; e $6,19 \%$ no grau leve. Não houve correlação entre ocorrência e severidade defluorose com o grau de satisfação estético tanto no sexo masculino como no feminino.

M attos et al. ${ }^{17}$ avaliaram a gravidade da fluorose endêmica e o comprometimento estético e funcional em indivíduos de sete a 22 anos de comunidades rurais de São Francisco (M G). A categoria TF = 1 a 3 foi observada em 37,5\% dos dentes; a TF $=4 / 5$, em $16 \%$ dos dentes e TF > 6 , em $28,4 \%$. Quanto à percepção do problema, os examinados relataram desconhecimento da causa real da fluorose, prejuízos na convivência social e o desejo de ter os dentes brancos.

Oliveira-Junior ${ }^{18}$ utilizou um índice de D ean. H ouve prevalência de $22,47 \%$ de fluorose dentária, com predomínio dos graus muito leve e leve. Os indivíduos mais jovens apresentaram maior prevalência, sendo esta de $32,4 \%$ e $16,8 \%$ para as idades de doze e quinze anos, respectivamente.

Carval ho et al. ${ }^{19}$ observaram a prevalência de fluorose dentaria efatores causais ou de risco em Aracaju (SE). A prevalência de fluorose dentária foi de 7,8\%, não implicando risco à saúde publica; porém, estudos semelhantes devem ser realizados para orientação de programas de educação em saúde bucal, tendo em vista que fatores de risco à fluorose foram detectados na população.

Deschamps e Sousa ${ }^{20}$ avaliaram a percepção da aparência dos dentes, prevalência e severidade da fluorose dentária. Para isso, analisaram 188 escolares de doze anos de idade. Constatou-se que $67 \%$ dos adolescentes apresentavam fluorose, sendo que $12,8 \%$ não estavam satisfeitos com a aparência. Não houve associação da insatisfação com a presença da fluorose. Entretanto, houve maior insatisfação entre aqueles que apresentaram os graus 4 e 5 , comparados com os níveis mais baixos.

Ditterich et al. ${ }^{21}$ verificaram autopercepção da fluorose dentária e sua relação com a satisfação com a aparência dos dentes. 0 estudo foi realizado com 473 escolares de dozeanos deidade, aplicando um questionário parcialmente estruturado. A prevalência de fluorose dentária foi de $19,65 \%$, distribuída no grau muito leve (12,9\%); leve $(5,9 \%)$ e moderado $(0,85 \%)$. As manchas provocadas pela fluorose dentária foram autopercebidas pelos escolares; porém, não contribuíram com o motivo de insatisfação com a aparência dental.

Hilgenberg et al. ${ }^{22}$ avaliaram a prevalência ea gravidade da fluorose dentária em 473 escolares 
de doze anos de idade e sua relação com a ingestão de fluoretos durante a infância. Através de um questionário, verificou-se a ingestão de fluoretos. A prevalência de fluorose dentária foi de $19,65 \%$, distribuída no grau muito leve ( $12,9 \%)$; leve $(5,9 \%)$ e moderado $(0,85 \%)$. N enhuma associação foi encontrada entre a presença defluorose e as variáveis: local de nascimento, tipo de água consumida, uso de dentifrício, quantidade dispensada de creme dental na parte ativa da escova, relato de ingestão proposital de dentifrício, realização de bochechos com flúor e número de escovações dentais diárias.

Rodrigues et al. ${ }^{23}$ verificaram os níveis de fluoreto no leite materno humano e a relação com os hábitos alimentares e de higiene das lactantes, estimando o potencial de risco de fluorose nos lactentesatravés da amamentação. Utilizou-seum questionário padronizado analisando variáveis como: uso de fluoretos, fonte da água consumi$\mathrm{da}$, bebidas, alimentos naturais e industrializados. Encontrou-se no leite materno valores entre 0,037 a 0,1837 ppm F-, com média de 0,095 ppm F-. Os níveis de fluoreto no leite materno não constituíram, individualmente, um fator de risco à fluorose, também não sendo possível observar relação entre os níveis de fluoreto presentes no leite e as informações obtidas através das mães.

Sartori et al. ${ }^{24}$ observaram a prevalência da cárie dentaria, necessidade detratamento ea prevalência da fluorose dentária em 309 escolares de doze anos de Indaiatuba (SP). 0 percentual de escolares livres de cáriefoi de 38,8\%. 0 Índicede Fluorose da Comunidade foi de 0,36. A prevalência de fluorose foi de $21,7 \%$, variando entreos graus muito leve a moderado.

\section{M étodo}

A partir de buscas em bases eletrônicas (Scielo, LiLacs, BBO), foram localizados estudos epidemiológicos que abordam a fluorose no Brasil. 0 critério deinclusão para figurar na presente análise foi apresentar dados primários de fluorose dentária em crianças/adolescentes em inquéritos epidemiológicos de delineamento transversal.

Os descritores de assunto foram: epidemiologia, fluorose dentária, crianças. Também foi utilizado 0 agrupamento destes descritores e intersecção com o país e ano de publicação. Foram selecionados artigos a partir de 1993, ano da realização da 2a Conferência Nacional deSaúde Bucal, cujo relatório el encava os vergonhosos indicadores de saúde e de morbidade bucal existentes, traduzidos pelos elevados índices de mutilações, cáries dentárias, doenças periodontais, câncer bucal, má oclusão e anomalias congênitas que colocam o Brasil entre os países de piores condições de saúde bucal no mundo ${ }^{25}$.

0 ano de 2004 delimita o fim do período de busca de artigos publicados, marcado como 0 ano de realização da 3a Conferência Nacional de Saúde Bucal, cujo relatório final recomenda "a implementação do SISÁGUA com a disponibilização das informaç̧ões para toda população brasileira e de alternativas viáveis para os casos de fluorose" ${ }^{\prime 26}$, refletindo a mudança na ênfase conferida a esta alteração de esmalte.

Com relação ao período subsequente à realização da 3a Conferência Nacional de Saúde Bucal, foi realizada a busca de resumos apresentados nas reuniões anuais da Sociedade Brasileira de Pesquisa O dontológica, nos anos 2005 e 2006.

A escolha recaiu sobre os resumos publicados no periódico Brazilian Oral Research, não tendo sido considerados outros estudos publicados em periódicos brasileiros, devido ao tempo que decorre entre a realização da pesquisa e sua efetiva publicação.

Dessa forma, não seria possível assegurar que a pesquisa científica publicada após 2004 tenha sido de fato produzida após a 3 a Conferência Nacional de Saúde Bucal.

Algumas limitações decorrem desta opção metodológica; contudo, para efeito da reflexão que se pretende efetuar no presente artigo, o parâmetro utilizado visa a evitar imprecisões.

\section{Discussão}

Segundo o Quadro 1, houve uma ampla abrangência dos estudos epidemiológicos de fluorose realizados no Brasil, em artigos publicados no período 1993-2004. Como se pode observar, houveum predomínio de estudos na Região Sudeste, especialmente no Estado de São Paulo. 0 maior número de trabalhos neste estado pode ser explicado pela alta concentração defaculdades deodontologia em São Paulo, fato este referido também por M oreira ${ }^{27} \mathrm{eN}$ arvai eAlmeida ${ }^{28}$. A faixa etária analisada variou de vinte meses a dezenove anos de idade. N ota-se ainda neste quadro uma maior prevalência defluorosenos graus mais leves, como observado por outros autores 4,10,13,18,22.

Em relação à fluoretação das águas de abastecimento público, o Parlamento brasileiro tomou importante decisão em 2004: analisou e rejeitou um projeto de lei que pretendia interrom- 
per a fluoretação das águas no país ${ }^{29}$. Segundo Kalamatianos e $\mathrm{Narvai}{ }^{30}$, a fluoretação da água foi considerada medida de saúde pública equânime, além de apresentar uma relação custo-benefício muito favorável. Frias ${ }^{31}$ constatou que a fluoretação da água apresentou um custo médio de $R \$ 0,08$ per capita/ano na cidade de São Paulo, em 2003, indicando uma economia de custos da ordem de $R \$ 348,68$ no serviço privado e de $R \$$ 83,68 no serviço público, por habitante/ano, para o grupo etário de sete a doze anos. Nos estudos que questionaram sobre a autopercepção da fluorose, os entrevistados não relataram percepção.
Cunha e Tomita ${ }^{32}$ relataram não encontrar, no Brasil, estudos longitudinais que esclareçam a mudança no perfil epidemiológico da fluorose, e comprovações científicas quejustifiquem alguma decisão no sentido de interromper a fluoretação das águas são escassas e/ou pouco consistentes.

O Quadro 2 mostra produção científica recente apresentada nos encontros da Sociedade Brasileira de Pesquisa Odontológica, no período 20052006. 0 público-alvo destes resumos tinha idade variando de cinco a 22 anos. Alguns estudos avaliaram a autopercepção da fluorose, a qual não teve relação com a sua presença na maioria dos

Quadro 1. Características de estudos epidemiológicos de fluorose no Brasil, em artigos publicados no período $1993-2004$.

\begin{tabular}{|c|c|c|c|c|}
\hline Autor e ano & Local & Idade & $\begin{array}{l}\text { Variáveis } \\
\text { de estudo }\end{array}$ & Resultados \\
\hline Paiva et al. (1993) & Urussanga (SC) & 5 a 10 & Fluorose & -- \\
\hline Tomita et al. (1995) & Piratininga (SP) & 6 a 14 & $\begin{array}{l}\text { Teor de flúor } \\
\text { Fluorose }\end{array}$ & $\begin{array}{l}\text { 1,05 em } 1991 \text { e 0,72ppm em 9234,44\% em } \\
\text { níveis "muito leve" e "moderado" }\end{array}$ \\
\hline Campos et al. (1998) & Brasília (DF) & 8 a 12 & Fluorose & $14,64 \%$ grau de fluorose variando de 1 a 3 \\
\hline M endonça et al. (1998) & $\begin{array}{l}\text { Belo Horizonte } \\
\text { (MG) }\end{array}$ & 7 e 10 & $\begin{array}{l}\text { Fluorose } \\
\text { Cárie }\end{array}$ & $\begin{array}{l}\text { 82,71\% fluorose "leve" Tendência de proteção } \\
\text { da fluorose contra a cárie }\end{array}$ \\
\hline Ribas et al. (1999) & São Paulo (SP) & 6 e 12 & Fluorose & $\begin{array}{l}29,47 \%(71,78 \%-T F=1 ; 21,47 \%-T F=2 ; \\
6,75 \%-T F=3)\end{array}$ \\
\hline Cangussu et al. (2001) & Itatiba (SP) & 5,12 e 15 & Fluorose & "muito leve" foi mais frequente \\
\hline Dini et al. (2000) & $\begin{array}{l}\text { Araraquara (SP) e } \\
\text { Américo Brasiliense } \\
(\mathrm{SP})\end{array}$ & 9 e 10 & Fluorose & $9,3 \%$ \\
\hline Forte et al. (2001) & Princesa I sabel (PB) & 10 a 15 & Fluorose & $20 \%$ apresentaram fluorose $(70 \% \quad T F=1)$ \\
\hline Oliveira e M ilbourne (2001) & Rio de Janeiro (RJ) & 7 e 12 & Fluorose & $7,9 \%$ apresentaram fluorose $(77 \% \quad$ TF $=1)$ \\
\hline Silva e M altz (2001) & Porto Alegre (RS) & 12 & Fluorose & $45,9 \% \mathrm{TF}=1,6,1 \% \mathrm{TF}=2$ e $0,9 \% \mathrm{TF}=3$ \\
\hline Alves et al. (2002) & Marília (SP) & 3 a 6 & Fluorose & $\begin{array}{l}92 \% \text { normal, } 3,8 \% \text { questionável, } 2,8 \% \text { muito } \\
\text { leve, 0,9\% leve, 0,3\% moderado, e 0,2\% } \\
\text { severa }\end{array}$ \\
\hline M enezes et al. (2002) & Piracicaba (SP) & 10 a 14 & $\begin{array}{l}\text { Autopercepção } \\
\text { da fluorose } \\
\text { Fluorose }\end{array}$ & $\begin{array}{l}\text { N ão houve percepção } \\
72 \%\end{array}$ \\
\hline Cypriano et al. (2003) & Piracicaba (SP) & 5 e 6 & $\begin{array}{l}\text { Fluorose } \\
\text { Cárie }\end{array}$ & $\begin{array}{l}\text { "muito leve" foi mais frequente } \\
\text { Houve redução de cárie com aumento da } \\
\text { fluorose }\end{array}$ \\
\hline Cangussu et al. (2004) & Salvador (BA) & 12 a 15 & Fluorose & $\begin{array}{l}29,6 \% \text { (prevalência); predomínio da categoria } \\
\text { "muito leve" }\end{array}$ \\
\hline
\end{tabular}


trabalhos. Os graus de fluorose mais prevalentes foram os mais leves. E embora se avente a possibilidade de um comprometimento estético relevante, não é o que se observa quando se faz uma avaliação que vá além dos aspectos normativos ${ }^{32}$.

No Q uadro 3, observa-se que a maioria dos estudos no período 1993-2004 utilizou o teste estatístico qui-quadrado. Analisando a origem do primeiro autor dos artigos selecionados, vêse um predomínio de faculdades públicas, com treze representantes e faculdades particulares apresentando quatro autores. A escassez da produção científica em universidades privadas pode ser resultado das políticas acadêmicas que resul-

Quadro 2. Características de estudos epidemiológicos de fluorose no Brasil, em resumos publicados nos anais da SBPqO, no período 2005-2006.

\begin{tabular}{|c|c|c|c|c|}
\hline Autor e ano & Local & Idade & Variáveis de estudo & Resultados \\
\hline Balen et al. (2005) & -- & -- & $\begin{array}{l}\text { Fatores de risco à } \\
\text { fluorose } \\
\text { Autopercepção } \\
\text { Fluorose }\end{array}$ & $\begin{array}{l}\text { Quantidade de creme dental } \\
\text { Não houve relação entre a percepção e } \\
\text { a presença de fluorose } \\
\text { M oderada e baixa severidade }\end{array}$ \\
\hline Kern et al. (2005) & Ponta Grossa (PR) & 12 & $\begin{array}{l}\text { Autopercepção da } \\
\text { fluorose }\end{array}$ & $\begin{array}{l}\text { Não houve correlação entre ocorrência } \\
\text { de fluorose e o grau de satisfação } \\
\text { estética }\end{array}$ \\
\hline Mattos et al. (2005) & $\begin{array}{l}\text { São Francisco } \\
\text { (MG) }\end{array}$ & 7 a 22 & $\begin{array}{l}\text { Fluorose } \\
\text { Autopercepção }\end{array}$ & $\begin{array}{l}\text { TFla3=37,5\% TF4/ } \\
5=16 \% \text { TF }>6=28,4 \% \\
\text { Prejuízos na convivência social e } \\
\text { desejo de ter dentes brancos }\end{array}$ \\
\hline $\begin{array}{l}\text { Oliveira-Júnior et al. } \\
\text { (2005) }\end{array}$ & Salvador (BA) & 12 e 15 & Fluorose & $\begin{array}{l}22,47 \% \text {, sendo } 32,4 \% \text { aos } 12 \text {, e } 16,8 \% \\
\text { aos } 15, \text { com predomínio dos graus } \\
\text { "muito leve" e "leve" }\end{array}$ \\
\hline Carvalho et al. (2006) & Aracaju (SE) & escolares & $\begin{array}{l}\text { Fluorose } \\
\text { Nunca recebeu } \\
\text { aplicação tópica de } \\
\text { flúor }\end{array}$ & $\begin{array}{l}7,8 \% \\
84,8 \%\end{array}$ \\
\hline Deschamps e Sousa (2006) & Piracicaba (SP) & 12 & $\begin{array}{l}\text { Autopercepção da } \\
\text { fluorose }\end{array}$ & $\begin{array}{l}\text { Não houve associação da insatisfação } \\
\text { com a presença de fluorose, apenas } \\
\text { nos casos mais severos }\end{array}$ \\
\hline Ditterich et al. (2006) & Ponta Grossa (PR) & 12 & $\begin{array}{l}\text { Autopercepção da } \\
\text { fluorose }\end{array}$ & $\begin{array}{l}\text { H ouve, porém sem insatisfação com a } \\
\text { aparência dental }\end{array}$ \\
\hline Hilgenberg et al. (2006) & Ponta Grossa (PR) & 12 & $\begin{array}{l}\text { Fluorose } \\
\text { Ingestão de fluoretos }\end{array}$ & $\begin{array}{l}\text { 12,9\% "muito leve", 5,9\% “leve", } \\
\text { 0,85\% "moderado" } \\
\text { Não houve relação entre ingestão de } \\
\text { fluoretos e presença de fluorose }\end{array}$ \\
\hline Rodrigues et al. (2006) & Pelotas (RS) & lactantes & $\begin{array}{l}\text { Teor de flúor no leite } \\
\text { materno } \\
\text { Risco de Fluorose }\end{array}$ & $\begin{array}{l}\text { Variou de } 0,037 \text { a } 0,1837 p p m F- \\
N \text { ão constitui, individualmente, um } \\
\text { fator de risco à fluorose }\end{array}$ \\
\hline Sartori et al. (2006) & Indaiatuba (SP) & 12 & $\begin{array}{l}\text { Cárie } \\
\text { Fluorose }\end{array}$ & $\begin{array}{l}61 \% ; \\
21,7 \% \text { de "muito leve" a "moderado" }\end{array}$ \\
\hline
\end{tabular}


tam em baixo incentivo à pesquisa, segundo M oreira ${ }^{27}$. Destes estudos, somente três receberam financiamento, sendo dois pelo CN Pq eum pela Fundação Cearense de A poio à Pesquisa. 0 financiamento de pesquisas na área de saúde bucal coletiva ainda mostra-se escasso, fato notado no estudo de Cormack eSilva Filho ${ }^{33}$, quan- do comparado às pesquisasqueenvolvem as ciências biológicas básicas, estudo de materiais e equipamentos odontológicos.

Analisando 0 Quadro 4, observa-se que todos os estudos do período 2005-2006 utilizaram 0 teste estatístico qui-quadrado. Os testes estatísticos, além de servirem para apresentação e

Quadro 3. Características de estudos epidemiológicos de fluorose no Brasil, em artigos publicados no período 1993-2004.

\begin{tabular}{|c|c|c|c|c|c|}
\hline Autor e ano & Local & Revista & $\begin{array}{l}\text { Origem do } \\
\text { primeiro autor }\end{array}$ & Financiamento & Teste estatístico \\
\hline Paiva et al. (1993) & Urussanga (SC) & Revista de Odontoped. & UFM G & -- & -- \\
\hline Tomita et al. (1995) & Piratininga (SP) & Rev. ABO Nacional & FOB-USP & -- & Kruskal-Wallis \\
\hline Campos et al. (1998) & Brasília (DF) & Rev. de Odontologia USP & $A B O-D F$ & -- & Qui-quadrado \\
\hline $\begin{array}{l}\text { M endonça et al. } \\
\text { (1998) }\end{array}$ & $\begin{array}{l}\text { Belo Horizonte } \\
\text { (MG) }\end{array}$ & RPG & UFMG & CNPq & $\begin{array}{l}\text { Qui-quadrado e } \\
\text { Pearson }\end{array}$ \\
\hline Ribas et al. (1999) & São Paulo (SP) & $\begin{array}{l}\text { Rev. O dontologia Univ. } \\
\text { Santo Amaro }\end{array}$ & $\begin{array}{l}\text { Universidade de } \\
\text { Santos }\end{array}$ & -- & -- \\
\hline Dini et al. (2000) & $\begin{array}{l}\text { Araraquara e } \\
\text { Américo } \\
\text { Brasiliense (SP) }\end{array}$ & British Dental Journal & $\begin{array}{l}\text { UNESP- } \\
\text { Araraquara-SP }\end{array}$ & -- & Qui-quadrado \\
\hline Cangussu et al. (2001) & Itatiba (SP) & $\begin{array}{l}\text { Cad. Saúde Pública, Rio de } \\
\text { Janeiro }\end{array}$ & $\begin{array}{l}\text { Fac. De } \\
\text { Odontologia }\end{array}$ & -- & $\begin{array}{l}\text { Qui-quadrado e } \\
\text { ANOVA }\end{array}$ \\
\hline Forte et al. (2001) & $\begin{array}{l}\text { Princesa Isabel } \\
\text { (PB) }\end{array}$ & $\begin{array}{l}\text { Pesquisa Odontológica } \\
\text { Brasileira }\end{array}$ & $\begin{array}{l}\text { UFBA } \\
\text { Univ. Federal de } \\
\text { Santa Catarina }\end{array}$ & -- & -- \\
\hline $\begin{array}{l}\text { Oliveira e M ilbourne } \\
(2001)\end{array}$ & $\begin{array}{l}\text { Rio de Janeiro } \\
\text { (RJ) }\end{array}$ & $\begin{array}{l}\text { Rev. Saúde Pública FSP- } \\
\text { U SP }\end{array}$ & UERJ & -- & M ann-W hitney \\
\hline Silva e M altz (2001) & $\begin{array}{l}\text { Porto Alegre } \\
\text { (RS) }\end{array}$ & $\begin{array}{l}\text { Pesquisa Odontológica } \\
\text { Brasileira }\end{array}$ & $\begin{array}{l}\text { Fac. de } \\
\text { Odontologia } \\
\text { UFRGS }\end{array}$ & -- & kappa \\
\hline Alves et al. (2002) & M arília (SP) & Rev. FOB-USP & UNIMAR & -- & Qui-quadrado \\
\hline M enezes et al. (2002) & Piracicaba (SP) & $\begin{array}{l}\text { Rev. Saúde Pública FSP- } \\
\text { USP }\end{array}$ & $\begin{array}{l}\text { Univ. Federal } \\
\text { do Ceará }\end{array}$ & $\begin{array}{l}\text { Fundação } \\
\text { Cearense de } \\
\text { Apoio à } \\
\text { Pesquisa }\end{array}$ & Qui-quadrado \\
\hline Cypriano et al. (2003) & Piracicaba (SP) & $\begin{array}{l}\text { Rev. Saúde Pública FSP- } \\
\text { USP }\end{array}$ & $\begin{array}{l}\text { FOP- } \\
\text { UNICAMP }\end{array}$ & -- & -- \\
\hline Cangussu et al. (2004) & Salvador (BA) & $\begin{array}{l}\text { Cad. Saúde Pública, Rio de } \\
\text { Janeiro }\end{array}$ & $\begin{array}{l}\text { Fac. De } \\
\text { Odontologia } \\
\text { UFBA }\end{array}$ & -- & Qui-quadrado \\
\hline
\end{tabular}


descrição dos achados, permitem a realização de testes de significância, importantes para confirmar, em termos de precisão estatística, os resultados verificados empiricamente. A respeito da origem do autor, observa-se uma predominância de faculdades públicas, assim como no Q uadro 3. Com relação ao financiamento, apenas três receberam auxílio para desenvolvimento do projeto de pesquisa, oriundo daCAPES $(n=2)$ e $\mathrm{CNPq}(\mathrm{n}=1)$.

Deve-se ressaltar que o relatório da 3 a Conferência Nacional de Saúde Bucal faz referência a garantir mais recursos para pesquisas, bolsas e projetos, relacionados com a área desaúdebucal coletiva, junto ao CN Pq (Conselho Nacional de Desenvolvimento Científico e Tecnológico), CAPES (Coordenação de Aperfeiçoamento de Pessoal de Ensino Superior) eDECIT (D epartamento deCiência e Tecnologia do M inistério da Saúde) de acordo com uma agenda prioritária de ações voltadas para 0 desenvolvimento do SUS, em todos os níveis ${ }^{26}$.
Os artigos no período 1993-2004 apresentados no Quadro 5 são transversais, com dados coletados em escolas públicas e particulares, e um em áreas urbanas.

No Quadro 6, vêse que os estudos do período 2005-2006 foram realizados em escolas públicas; porém, houve estudo realizado em casas, hospital e escolas particulares.

Segundo Cunha e Tomita ${ }^{32}$, o delineamento dos diferentes estudos disponíveis na literatura nacional mostra diversidades quanto ao planejamento amostral, tamanho da amostra, faixa etária, confiabilidade dos dados segundo os diferentes examinadores, reprodutibilidade do estudo e fidedignidade dos dados de vigilância sanitária, bem como a base territorial abordada.

A maioria dos estudos concentra-se em áreas urbanas e a predominância no Brasil dos graus "muito leve" e "leve" de fluorose mostra não haver comprometimentos de ordem funcional. A baixa percepção da fluorose pela população, alia-

Quadro 4. Características de estudos epidemiológicos de fluorose no Brasil, em resumos publicados nos anais da SBPqO, no período 2005-2006.

\begin{tabular}{|c|c|c|c|c|c|}
\hline Autor e ano & Local & Revista & $\begin{array}{l}\text { Origem do } \\
\text { primeiro autor }\end{array}$ & Financiamento & Teste estatístico \\
\hline Balen et al. (2005) & -- & Brazilian Oral Research & $\begin{array}{l}\text { Univ. Luterana do } \\
\text { Brasil }\end{array}$ & -- & Qui-quadrado \\
\hline Kern et al. (2005) & Ponta Grossa (PR) & Brazilian Oral Research & $\begin{array}{l}\text { Univ. Estadual de } \\
\text { Ponta Grossa }\end{array}$ & -- & Qui-quadrado \\
\hline M attos et al. (2005) & São Francisco (M G) & Brazilian Oral Research & $\begin{array}{l}\text { Univ. Federal de } \\
\text { Minas Gerais }\end{array}$ & -- & -- \\
\hline $\begin{array}{l}\text { Oliveira-Júnior et al. } \\
\text { (2005) }\end{array}$ & Salvador (BA) & Brazilian Oral Research & $\begin{array}{l}\text { Univ. Federal da } \\
\text { Bahia }\end{array}$ & -- & -- \\
\hline Carvalho et al. (2006) & Aracaju (SE) & Brazilian Oral Research & Univ. Tiradentes & CNPq & -- \\
\hline $\begin{array}{l}\text { Deschamps e Sousa } \\
\text { (2006) }\end{array}$ & Piracicaba (SP) & Brazilian Oral Research & $\begin{array}{l}\text { Univ. Estadual de } \\
\text { Campinas }\end{array}$ & -- & Qui-quadrado \\
\hline $\begin{array}{l}\text { Ditterich et al. } \\
\text { (2006) }\end{array}$ & Ponta Grossa (PR) & Brazilian Oral Research & $\begin{array}{l}\text { Univ. Estadual de } \\
\text { Ponta Grossa }\end{array}$ & CAPES & Qui-quadrado \\
\hline $\begin{array}{l}\text { Hilgenberg et al. } \\
\text { (2006) }\end{array}$ & Ponta Grossa (PR) & Brazilian Oral Research & $\begin{array}{l}\text { Univ. Estadual de } \\
\text { Ponta Grossa }\end{array}$ & CAPES & Qui-quadrado \\
\hline $\begin{array}{l}\text { Rodrigues et al. } \\
(2006)\end{array}$ & Pelotas (RS) & Brazilian Oral Research & $\begin{array}{l}\text { Univ. Federal de } \\
\text { Pelotas }\end{array}$ & -- & -- \\
\hline Sartori et al. (2006) & Indaiatuba (SP) & Brazilian Oral Research & Univ. Paranaense & -- & -- \\
\hline
\end{tabular}


da à sua baixa prevalência, evoca o necessário de bate sobre as questões de saúde coletiva no país.

O período 1993-2004 demarca o intervalo de tempo decorrido entre a 2 a Conferência $\mathrm{N}$ acional de Saúde Bucal e a $3^{a}$ Conferência Nacional de Saúde Bucal. No período subsequente, 20052006, a busca de dados primários apresentados em reuniões científicas confirmou os achados existentes na literatura.
Visto quea produção científica nacional constitui uma importante fonte de conhecimentos para subsidiar a elaboração de políticas públicas para o setor saúde, os sucessivos e pontuais estudos analisados mostram que, no que diz respeito à fluorose, o diagnóstico epidemiológico reafirma a necessidade, a importância e a segurança da fluoretação das águas de abastecimento público como medida de saúde coletiva.

Quadro 5. Características de estudos epidemiológicos de fluorose no Brasil, em artigos publicados, no período $1993-2004$.

\begin{tabular}{|c|c|c|c|c|}
\hline Autor e ano & Tipo de estudo & Dados & Fonte dos dados e forma de coleta & Local do estudo \\
\hline Paiva et al. (1993) & Estudo transversal & Primários & $\begin{array}{l}\mathrm{N}=299 \text {, entre } 5 \text { e } 10 \text { anos (exame } \\
\text { clínico) }\end{array}$ & Escolas \\
\hline Tomita et al. (1995) & Estudo transversal & Primários & $\mathrm{N}=270$, de 6 a 14 anos (exame clínico) & Escolas Públicas \\
\hline Campos et al. (1998) & Estudo transversal & Primários & $\mathrm{N}=833$, de 8 a 12 anos (exame clínico) & Escolas Públicas \\
\hline M endonça et al. (1998) & Estudo transversal & Primários & $\mathrm{N}=797$, de 7 e 10 anos (exame clínico) & Escolas \\
\hline Ribas et al. (1999) & Estudo transversal & Primários & $\mathrm{N}=553$, de 6 aos 12 anos (exame clínico) & Escola Particular \\
\hline Dini et al. (2000) & Estudo transversal & Primários & $\mathrm{N}=497$, de 9 e 10 anos (exame clínico) & Escolas Públicas \\
\hline Cangussu et al. (2001) & Estudo transversal & Primários & $\begin{array}{l}\mathrm{N}=252 \text {, de } 5,12 \text { e } 15 \text { anos (exame } \\
\text { clínico) }\end{array}$ & $\begin{array}{l}\text { Diferentes áreas } \\
\text { urbanas }\end{array}$ \\
\hline Forte et al. (2001) & Estudo transversal & Primários & $\mathrm{N}=142$, de 10 a 15 anos (exame clínico) & Escolas Públicas \\
\hline Oliveira e Milbourne (2001) & Estudo transversal & Primários & $\mathrm{N}=553$, de 6 aos 12 anos (exame clínico) & Escolas Públicas \\
\hline Silva e M altz (2001) & Estudo transversal & Primários & $\begin{array}{l}\mathrm{N}=1000,12 \text { anos (exame clínico e } \\
\text { questionário) }\end{array}$ & $\begin{array}{l}\text { Escolas Públicas } \\
\text { e Particulares }\end{array}$ \\
\hline Alves et al. (2002) & Estudo transversal & Primários & $\mathrm{N}=994$, de 3 aos 6 anos (exame clínico) & Escolas Públicas \\
\hline M enezes et al. (2002) & Estudo transversal & Primários & $\begin{array}{l}\mathrm{N}=57 \text {, de } 10 \text { aos } 14 \text { anos (exame clínico } \\
\text { e questionário) }\end{array}$ & Escola Pública \\
\hline Cypriano et al. (2003) & Estudo transversal & Primários & $\mathrm{N}=2805$, de 5 e 6 anos (exame clínico) & Escolas Públicas \\
\hline Cangussu et al. (2004) & Estudo transversal & Primários & $\begin{array}{l}\mathrm{N}=3.313 \text {, de } 12 \text { e } 15 \text { anos (exame clínico } \\
\text { e questionário) }\end{array}$ & $\begin{array}{l}\text { Escolas Públicas } \\
\text { e Particulares }\end{array}$ \\
\hline
\end{tabular}




\section{Colaboradores}

NE Tomita participou da concepção teórica ediscussão. BSA Barros participou do levantamento de dados e redação.

\section{Referências}

1. Paiva SM, Barros F, Manoel A. Contribuição ao estudo da fluorose dentária, na dentição permanente, numa comunidade com fluorose endêmica (Cocal-U russanga- Santa Catarina). Rev Odontopediatr 1993; 2:5-12.

2. Tomita NE, Panighel CPMA, Narvai PC, Lopes ES. Implicações da vigilância à saúde sobre a ocorrência de fluorose dental. Revista $A B O \mathrm{Nacional}$ 1995; 3:318-23.

3. Campos DL, Farias DG, Toledo AO, Bezerra ACB. Prevalência de fluorose dentária em escolares de Brasília - Distrito Federal. Rev Odontol Univ São Paulo 1998; 12:225-230.

4. M endonça LL, Kirchner UL, Costa RN, Giovannini JFBG, Pinto MR, Luz MAR. Estudo multicentro da fluorose dental e cárie dental em escolares de 7 e 10 anos de Belo Horizonte. Parte I. RPG: Rev Pós-grad 1998; 5:101-107.

Quadro 6. Características de estudos epidemiológicos de fluorose no Brasil, em resumos publicados nos anais da SBPqO, no período 2005-2006.

\begin{tabular}{|c|c|c|c|c|}
\hline Autor e ano & Tipo de estudo & Dados & Fonte dos dados e forma de coleta & Local do estudo \\
\hline Balen et al. (2005) & Estudo transversal & Primários & N = 162 (questionário para o responsável) & -- \\
\hline Kern et al. (2005) & Estudo transversal & Primários & $\begin{array}{l}\mathrm{N}=113,12 \text { anos (questionário } \\
\text { parcialmente estruturado) }\end{array}$ & Escolas públicas \\
\hline Mattos et al. (2005) & Estudo transversal & Primários & $\begin{array}{l}\mathrm{N}=214 \text {, de } 7 \text { a } 22 \text { anos (exame clínico e } \\
\text { entrevista) }\end{array}$ & Casas \\
\hline Oliveira-Júnior et al. (2005) & Estudo transversal & Primários & $\begin{array}{l}\mathrm{N}=2.110 \text {, de } 12 \text { a } 15 \text { anos (questionário } \\
\text { semi-estruturado e exame clínico) }\end{array}$ & Escolas \\
\hline Carvalho et al. (2006) & Estudo transversal & Primários & $\begin{array}{l}\text { N = } 369 \text { (questionário realizado com } \\
\text { escolares, e seus responsáveis) }\end{array}$ & Escolas públicas \\
\hline Deschamps e Sousa (2006) & Estudo transversal & Primários & $\begin{array}{l}\mathrm{N}=188,12 \text { anos (exame clínico em } \\
\text { escolares) }\end{array}$ & Escolas públicas \\
\hline Ditterich et al. (2006) & Estudo transversal & Primários & $\begin{array}{l}\mathrm{N}=473,12 \text { anos (questionário } \\
\text { parcialmente estruturado) }\end{array}$ & Escolas públicas \\
\hline Hilgenberg et al. (2006) & Estudo transversal & Primários & $\begin{array}{l}\mathrm{N}=473,12 \text { anos (questionário retrospectivo } \\
\text { e exames clínicos em escolares) }\end{array}$ & Escolas públicas \\
\hline Rodrigues et al. (2006) & Estudo transversal & Primários & N = 11 (questionário padronizado) & Hospital \\
\hline Sartori et al. (2006) & Estudo transversal & Primários & $\begin{array}{l}\mathrm{N}=309,12 \text { anos (exame clínico em } \\
\text { escolares) }\end{array}$ & Escolas públicas \\
\hline
\end{tabular}


5. Ribas TRC, Armonia PL, Saraceni G Jr, Campos PRB. Avaliação da fluorose dentária em escolares do Colégio Vicente de Paulo, situado na Zona Leste do M unicípio de São Paulo - 1999. Rev Odontol Univ St Amaro 1999; 4:62-65.

6. Dini EL, Holt RD, Bedi R. Prevalence of caries and developmental defects of enamel in 9-10 year old children living in areas in Brazil with differing water fluoride histories. Br Dent J. 2000; 188:146-149.

7. Cangussu MCT, Coelho EO, Fernandez RAC. Epidemiologia e iniquidade em saúde bucal aos 5,12 e 15 anos de idade no município de Itatiba, São PauIo, 2000. Rev Fac Odontol Bauru 2001; 9:77-85.

8. Forte FDS, Freitas CH SM, Sampaio FC, Jardim MCAM . Fluorose dentária em crianças de Princesa Isabel, Paraíba. Pesqui Odontol Bras. 2001; 15:87-90.

9. Oliveira BHO, Milbourne P. Fluorose dentária em incisivos superiores permanentes em crianças de escola pública do Rio de Janeiro, RJ. Rev. Saude Publica 2001; 35:276-282.

10. Silva $B B, M$ altz $M$. Prevalência de cárie, gengivite e fluorose em escolares de 12 anos de Porto AlegreRS, Brasil, 1998/1999. Pesqui O dontol Bras 2001; 15:208-214.

11. Alves NC, Orenha ES, Reche NSG, Pelli MPS. Pre valência de fluorose dentária na dentição decídua em crianças participantes de programas de prevenção e promoção de saúde no município de Marília-SP. Rev Fac Odontol Bauru 2002; 10(3):156-163.

12. Menezes LMB, Sousa MLR, Rodrigues LKA, Cury JA. Autopercepção da fluorose pela exposição a flúor pela água e dentifrício. Rev. Saude Publica 2002; 36(6):752-754

13. Cypriano S, Sousa M LR, Rihs LB, Wada RS. Saúde bucal dos pré-escolares, Piracicaba, Brasil, 1999. Rev. Saude Publica 2003; 37:247-253.

14. Cangussu MCT, Fernandez RAC, Rivas CC, Ferreira C Jr, Santos LCS. Prevalência da fluorose dentária em escolares de 12 a 15 anos de idade em Salvador, Bahia, Brasil, 2001. Cad Saude Publica 2004; 20:129-135.

15. Balen EA, Celeste RK, Vecchia GFD, Cerutti D, Fontana-Júnior $A$. Fatores de risco associados à fluorose dental com uma população de baixa prevalência [abstract P0 024]. Braz Oral Res. 2005; 19:28.

16. Kern R, Ditterich RG, Virgens-Filho JS, Wambier DS, Romanelli M CM OV, Rodrigues CK. Correlação da autopercepção do manchamento e da aparência dental [abstract Pc227]. Braz Oral Res. 2005; 19:231.

17. M attos GCM, Ferreira EF, Vargas AM D, Castilho LS, M enegasse LN, Fantinel LM. Fluorose endêmica em São Francisco-M G: gravidade e comprometimento estético e funcional [abstract Ib066]. Braz Oral Res. 2005; 19:75.

18. Oliveira-Júnior SR, Cangussu MCT, Lopes LS, Soares $A P$, Ribeiro $A A$, Fonseca $L A$. Fluorose dentária em escolares de12 a 15 anos de idade, Salvador, Bahia, Brasil, 2004 [abstract Ib153]. Braz Oral Res. 2005; 19:86.

19. Carvalho RWF, Santos CNA, Oliveira CCC, Gonçalves SRJ. Estudo epidemiológico de fluorose dentária em Aracaju - SE [abstract PI019]. Braz Oral Res. 2006; 20:51.
20. Deschamps N, Sousa MLR. Percepção da fluorose dental segundo severidade em adolescentes de um município com água fluoretada [abstract Ib058]. Braz Oral Res. 2006; 20:96.

21. Ditterich RG, Portero PP, Pereira JLN, Daniel SCS, M oysés SJ, Wambier DS. Autopercepção da fluorose dentária em escolares de 12 anos de idade [abstract P0 090]. Braz Oral Res. 2006; 20:51.

22. Hilgenberg SP, Ditterich RG, Grau P, Romanelli M CM OV, M oysés SJ, Wambier DS. Prevalência de fluorose dentária e sua relação com a ingestão de fluoretos durante a infância em escolares de 12 anos [abstract P0 088]. Braz Oral Res.2006; 20:41.

23. Rodrigues $P B$, Oliveira LJC, Bolek RF, Silva VM, Lund RG, Pino FAB. Teor de flúor no leite materno: possibilidade de fluorose? Estudo preliminar [abstract Ic091]. Braz Oral Res. 2006; 20:131.

24. Sartori R, Albuquerque SC, Silva DD, Gomes VE, Rins LB, Sousa MLR, Cypriano S. Saúde bucal em escolares após 25 anos de fluoretação da água de abastecimento público [abstract PI007]. Braz Oral Res. 2006; 20:49.

25. Conferência Nacional de Saúde Bucal. Relatório Final. Brasília: Centro de Documentação do Ministério da Saúde; 1993.

26. Conferência Nacional de Saúde Bucal. Acesso e qualidade superando exclusão social. Relatório Final. Brasília: Centro de Documentação do M inistério da Saúde; 2004.

27. Moreira RF, Nico LS, Tomita NE, Ruiz T. A saúde bucal do idoso brasileiro: revisão sistemática sobre o quadro epidemiológico e acesso aos serviços de saúde bucal. Cad Saude Publica 2005; 21:1665-1675.

28. Narvai PC, Almeida ES. 0 sistema de saúde e as políticas de saúde na produção científica odontológica brasileira no período 1986-1993. Cad Saude Publica 1998; 14:513-521.

29. Narvai PC, Frazão P, Fernandez RAC. Fluoretação da água e democracia. Saneas 2004; 2(18):29-33.

30. Kalamatianos PA, Narvai PC. Aspectos éticos do uso de produtos fluorados no Brasil: uma visão dos formuladores de políticas públicas de saúde. Cien Saude Colet 2006; 11(1):63-69.

31. Frias AC. Custo-efetividade da fluoretação das águas de abastecimento público no município de São Paulo, no período de 1985-2003 [tese]. São Paulo (SP): Faculdade de Saúde Pública, U niversidade de São Paulo; 2004.

32. Cunha LF, Tomita NE. Fluorose dentária no Brasil: uma revisão sistemática do período 1993/2004. Cad Saude Publica 2006; 22(9):1809-1816.

33. Cormack EF, Silva CF Filho. A pesquisa científica odontológica no Brasil. Rev Assoc Paul Cir Dent 2000; 54:242-247.

Artigo apresentado em 04/12/2006

Aprovado em 18/05/2007 